\title{
Evaluation of a semi-automated platelet-counting system
}

\author{
R. M. ROWAN, C. FRASER, J. H. GRAY, AND G. A. MCDONALD
}

From the Department of Haematology, Royal Infirmary, Glasgow

SUMMARY Coulter Electronics Ltd have produced a semi-automated platelet-counting system. Platelet-rich plasma may be obtained either by tube sedimentation or by means of the Thrombofuge, the latter being an instrument designed to produce accelerated sedimentation. The instrument is linear over the entire range of platelet counts, and machine reproducibility is good. Comparison of machine-rated with visual counts satisfied statistical evaluation. The technique can be handled by one operator and platelet counts can be achieved at the rate of 30 per hour by both methods although individual counts on the Thrombo-fuge may be obtained in approximately one-quarter of the time required for tube sedimentation. The throughput using the Thrombo-fuge could certainly be doubled were two sample plates supplied.

Few problems were encountered during the evaluation and most could be avoided by meticulous technique. Visual counts must be performed when the sample haematocrit is greater than $50 \%$. Discrepant counts have been obtained in patients with white cell counts exceeding $50 \times 10^{9} / 1$ and in patients with giant platelets. ESR elevation for any reason does not lead to serious discrepancy in results. The incidence of platelet clumping due to the presence of platelet agglutinins and of microclot formation due to inadequate mixing is probably much higher than is commonly thought, and certainly peripheral blood film scrutiny should never be omitted in patients with low counts. Careful examination of peripheral blood films must be combined with instrument counting for some time lest further causes of discrepant counting emerge.

The Coulter Thrombocounter $\mathrm{C}$ is a special purpose version of the Coulter Counter, rationalised and simplified to count platelets, and comprises integral sampling and electronic assemblies. The orifice selected in this system has a 70 micron diameter. The orifice diameter is determined by three factors, namely, the range of platelet size, electronic and biological noise at the lower end of the system, and, at the other extreme, larger non-platelet particles. Thus the useful range of orifice is $4-40 \%$ of the total diameter. The threshold controls on the Thrombocounter $\mathrm{C}$ are pre-set for 3-30 cubic microns, figures which satisfy the physical requirements of a platelet-counting system. Up to 40000 particles may be counted during an eight-second cycle.

Since this is not a fully automatic process, separation of red cells from platelet-rich plasma must be effected as a first step. Platelet-rich plasma is prepared by a sedimentation technique which is

Received for publication 25 August 1976 dependent on platelets and red cells being negatively charged. During sedimentation red cells trap plasma which is platelet free, leading to an excess of platelets in the supernatant plasma. Therefore two corrections require to be made to achieve a whole blood platelet count, the first being a correction for excess platelets in supernatant plasma and the second for haematocrit (Bull et al., 1965). The correction for whole blood platelet count was originally made using a circular slide rule. On the up-dated version of the instrument-the Thrombocounter $\mathrm{C}$-however, the whole blood platelet count is obtained by dialling the haematocrit value into the system, the correction factor calculation for platelet-rich plasma being built into the instrument. Separation by sedimentation may be achieved either in small plastic tubes or by means of the Thrombofuge.

For tube sedimentation approximately $0.1 \mathrm{ml}$ whole blood, anticoagulated with EDTA, is aspirated into plastic sedimentation tubes and allowed to settle at an angle of $45^{\circ}$ for 30 minutes. The tube is 
then cut just above the sedimented red cells and platelet-rich plasma interface. A 3.3 microlitre pipette is filled with platelet-rich plasma by capillary attraction. This plasma is diluted in $10 \mathrm{ml}$ of Isoton (1 in 3000 dilution). The pipette should be rinsed three or four times into the Isoton which is then gently mixed. The dilution is now ready to be counted.

Alternatively, the Thrombo-fuge (Fig. 1), an electromechanical instrument designed to bring about accelerated sedimentation, may be used to effect separation. The sample tubes are one-third filled $(1 \mathrm{ml})$ with whole blood and placed in the sample plate with each tube tilted at $3^{\circ}$ from vertical. The Thrombo-fuge is then switched on and the sample plate spins at $880 \mathrm{rpm}$. As the plate spins, the red cells begin to stack together, separating from the plasma, the so-called rouleaux formation stage. This phase occurs within 20-45 seconds. At the end of the $\mathbf{4 5}$ seconds the sample motor is programmed to stop, leaving the sample plate spinning freely. The plate takes 60 seconds to come to rest. When the sample plate is at rest, the red cells reach the bottom of the tube and start to pack. Five minutes after switch-on the Thrombo-fuge buzzer sounds and the 'wait light' goes out, signifying the end of the sedimentation cycle. This simple instrument reduces the preparation of platelet-rich plasma samples to less than one-sixth of the time previously required using free-standing tube sedimentation. A 6.6 microlitre pipette is filled from the mid-zone of the platelet-rich plasma and diluted in $20 \mathrm{ml}$ of Isoton ( 1 in 3000 dilution).

The haematocrit level is a limiting factor in the computation of the whole blood platelet count. Haematocrit levels of between $20 \%$ and $50 \%$ may be dialled into the machine for automatic calculation. For haematocrit levels between $10 \%$ and $20 \%$, whole blood platelet counts may be calculated using the charts in the instruction manual. Haematocrit levels greater than $55 \%$ constitute an absolute indication for manual counting. Although the instruction manual indicates that further dilution is necessary with machine counts greater than 24000 , this was found to be unnecessary in practice, dilution being required only with machine counts in excess of 30000 ( 1 in 6000 dilution). It is important to remember that when this is done the whole blood platelet count obtained must be multiplied by a factor of two. With machine counts of less than 1000

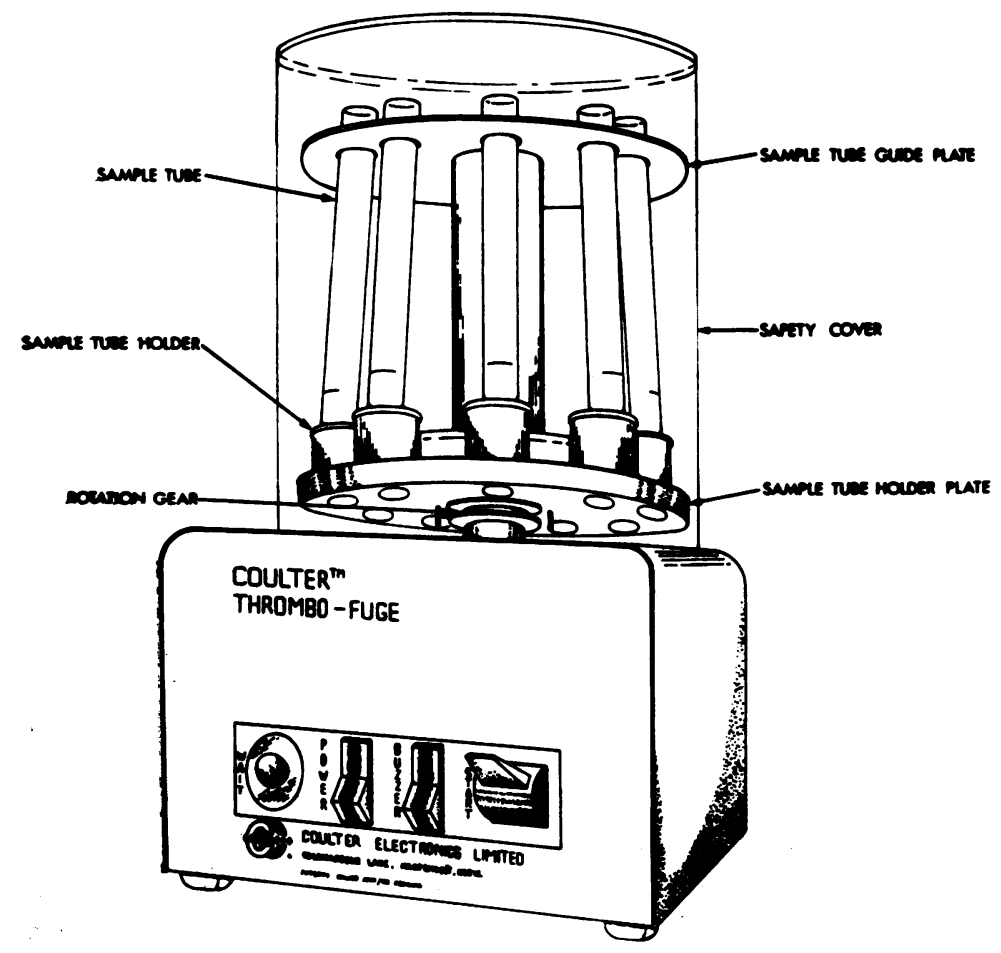

Fig. 1 The Thrombo-fuge (front view). 
concentration is necessary with appropriate arithmetical correction.

A certain minimum maintenance is necessary to ensure proper function of the Thrombocounter $\mathrm{C}$. This should be carried out on a regular basis and can be performed by any competent technician. No routine maintenance of the Thrombo-fuge is necessary other than occasional cleansing of the sample plate and the tube holder sockets.

\section{Material and methods}

The haematology department of a large teaching hospital was the site of this evaluation, blood being obtained from patients suffering from a wide variety of diseases. Sequestrene (final concentration $1 \mathrm{mg} / \mathrm{ml}$ ) was used as anticoagulant. All counts were performed within 3 hours of sampling. The visual direct platelet-counting technique using formal citrate diluent described by Dacie and Lewis (1975) was used. The number of platelets in one or more areas of $1 \mathrm{~mm}^{2}$ were counted, the total platelets counted always exceeding 100 . Duplicate visual counts were true, being performed by different experienced technicians.

The Thrombocounter $\mathrm{C}$ was set up in accordance with the makers' recommendations. The instruction manual is comprehensive and accurate. Operational problems were minimal.

\section{Results}

System evaluation was carried out to assess equipment performance and to compare two techniques of platelet-rich plasma preparation. Since there are known differences between the haematocrit as measured on the Coulter Model $\mathbf{S}$ and by manual methods, both techniques were used where appropriate. Significant differences were not noted in whole blood platelet counts with either technique of haematocrit measurement.

\section{INSTRUMENT ACCURACY}

Before evaluation, the Thrombocounter $\mathrm{C}$ was checked to ensure linearity. Four sequestrene samples were obtained through a platelet count range of $175 \times 10^{9} / 1$ to $700 \times 10^{9} / 1$, two samples being prepared by the Thrombo-fuge and two by tube sedimentation. Four dilutions were made from each sample using Isoton, representing $100 \%$, $75 \%, 50 \%$, and $25 \%$, and platelet counts were carried out (Fig. 2). As can be seen, in each case agreement throughout the entire range was without bias. Four further sequestrene samples of platelet level $20 \times 10^{9} / 1$ to $120 \times 10^{9} / 1$ were concentrated up to $600 \%$ by adding appropriate aliquots of

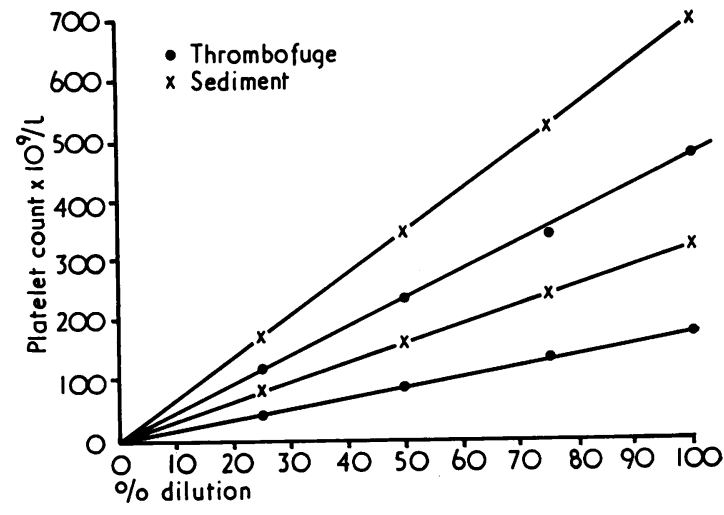

Fig. 2 Instrument linearity using dilution technique.

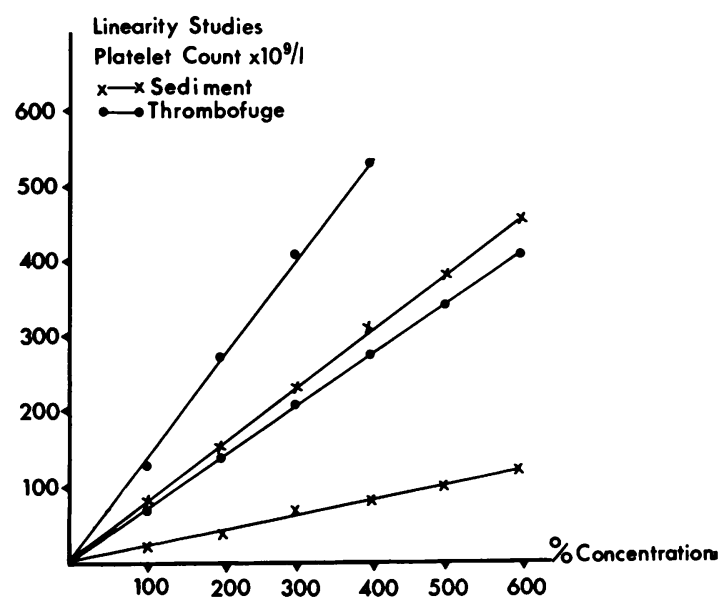

Fig. 3 Instrument linearity using concentration technique.

platelet-rich plasma (Fig. 3). Again linearity proved to be satisfactory.

To assess carry-over, a sample of platelet count $340 \times 10^{9} / 1$ was diluted with Isoton producing two series of five dilutions each ranging from $100 \%$ to $10 \%$. These dilutions were presented to the Thrombocounter $\mathrm{C}$ in the order shown in Table 1 . These results are not outwith the confidence limits defined in the linearity experiments. It can therefore be deduced that no significant carry-over occurs.

No machine drift was detected during any part of the evaluation.

INSTRUMENT PRECISION

Instrument precision was examined using replicate and duplicate studies. Eight blood samples of 
Table 1 Instrument carry-over: whole blood sample of platelet count $340 \times 10^{\circ} / \mathrm{l}$ diluted with Isoton, producing two series of five dilutions ranging from $100 \%$ to $10 \%$

\begin{tabular}{llll}
\hline Dilution \% & Predicted & Actual & $\%$ \\
\hline 10 & $36 \times 10 \% 1$ & $38(+2) \times 10 \%$ & +5.6 \\
25 & 90 & $90(0)$ & $-2 \cdot 2$ \\
50 & 180 & $176(-4)$ & $-1 \cdot 9$ \\
75 & 270 & $265(-5)$ & \\
100 & 360 & $265(-5)$ & $-1 \cdot 9$ \\
75 & 270 & $175(-5)$ & -2.8 \\
50 & 180 & $89(-1)$ & $-1 \cdot 1$ \\
25 & 90 & $37(+1)$ & +2.8 \\
10 & 36 & $358(-2)$ & -0.6 \\
100 & 360 & $37(+1)$ & +2.8 \\
10 & 36 & & \\
\hline
\end{tabular}

platelet count range $15 \times 10^{9} / 1$ to $698 \times 10^{9} / 1$ prepared by tube sedimentation were successively re-sampled 20 times (Table 2). In no replicate series did more than $5 \%$ of results exceed the two standard deviation range. Further replicate studies were carried out on seven blood samples of platelet count range $16 \times 10^{9} / 1$ to $528 \times 10^{9} / 1$, platelet-rich plasma being obtained by the Thrombo-fuge (Table $3)$. Only at the $16 \times 10^{9} / 1$ count did more than $5 \%$ of results fall outwith the two standard deviation range.

Duplicate studies were carried out on 68 blood samples, using the tube sedimentation technique,

Table 2 Platelet-rich plasma from eight blood samples prepared by tube sedimentation successively re-sampled $\times 20$

\begin{tabular}{|c|c|c|c|c|c|c|c|c|}
\hline No. & 1 & 2 & 3 & 4 & 5 & 6 & 7 & 8 \\
\hline $\begin{array}{r}1 \\
2 \\
3 \\
4 \\
5 \\
6 \\
7 \\
8 \\
9 \\
10 \\
11 \\
12 \\
13 \\
14 \\
15 \\
16 \\
17 \\
18 \\
19 \\
20\end{array}$ & $\begin{array}{l}17 \\
16 \\
14 \\
16 \\
16 \\
14 \\
14 \\
14 \\
14 \\
14 \\
16 \\
14 \\
16 \\
16 \\
16 \\
17 \\
16 \\
14 \\
16 \\
16\end{array}$ & $\begin{array}{l}62 \\
62 \\
65 \\
64 \\
65 \\
62 \\
62 \\
64 \\
65 \\
65 \\
64 \\
65 \\
67 \\
64 \\
64 \\
64 \\
58 \\
62 \\
65 \\
64\end{array}$ & $\begin{array}{l}145 \\
173 \\
157 \\
172 \\
223 \\
164 \\
187 \\
187 \\
161 \\
172 \\
163 \\
172 \\
178 \\
174 \\
183 \\
162 \\
173 \\
162 \\
167 \\
175\end{array}$ & $\begin{array}{l}255 \\
275 \\
265 \\
283 \\
280 \\
281 \\
277 \\
280 \\
280 \\
277 \\
283 \\
260 \\
170 \\
270 \\
265 \\
262 \\
260 \\
262 \\
260 \\
270\end{array}$ & $\begin{array}{l}364 \\
380 \\
358 \\
359 \\
369 \\
358 \\
376 \\
356 \\
378 \\
360 \\
368 \\
369 \\
360 \\
341 \\
367 \\
367 \\
386 \\
379 \\
354 \\
369\end{array}$ & $\begin{array}{l}468 \\
450 \\
404 \\
450 \\
492 \\
475 \\
470 \\
475 \\
492 \\
480 \\
484 \\
496 \\
462 \\
496 \\
472 \\
485 \\
496 \\
450 \\
492 \\
462\end{array}$ & $\begin{array}{l}572 \\
500 \\
572 \\
604 \\
624 \\
618 \\
618 \\
624 \\
630 \\
592 \\
604 \\
580 \\
572 \\
592 \\
568 \\
604 \\
560 \\
604 \\
620 \\
604\end{array}$ & $\begin{array}{l}742 \\
739 \\
689 \\
739 \\
694 \\
688 \\
716 \\
681 \\
681 \\
683 \\
685 \\
692 \\
685 \\
701 \\
681 \\
677 \\
706 \\
685 \\
677 \\
714\end{array}$ \\
\hline $\begin{array}{l}\text { Mean } \\
2 \text { SD Range } \\
\text { SE } \\
\text { Coeff. Var. }\end{array}$ & $\begin{array}{l}15 \cdot 3 \\
13 \cdot 1-17 \cdot 5 \\
0 \cdot 25 \\
7 \cdot 2\end{array}$ & $\begin{array}{l}63 \cdot 7 \\
59 \cdot 9-67 \cdot 5 \\
0 \cdot 42 \\
3 \cdot 0\end{array}$ & $\begin{array}{l}172 \cdot 5 \\
141 \cdot 1-203 \cdot 9 \\
3 \cdot 5 \\
9 \cdot 1\end{array}$ & $\begin{array}{c}270 \cdot 8 \\
252 \cdot 6-289 \\
2 \cdot 04 \\
3 \cdot 4\end{array}$ & $\begin{array}{l}365 \cdot 9 \\
344 \cdot 5-387 \cdot 3 \\
2 \cdot 39 \\
2 \cdot 9\end{array}$ & $\begin{array}{l}472 \cdot 6 \\
427 \cdot 6-517 \cdot 6 \\
5 \cdot 00 \\
4 \cdot 8\end{array}$ & $\begin{array}{l}593 \cdot 1 \\
532 \cdot 1-654 \cdot 1 \\
6 \cdot 80 \\
5 \cdot 1\end{array}$ & $\begin{array}{l}697 \cdot 8 \\
655-740 \cdot 6 \\
4 \cdot 80 \\
3 \cdot 1\end{array}$ \\
\hline
\end{tabular}

Table 3 Platelet-rich plasma from seven blood samples prepared using Thrombo-fuge successively re-sampled $\times 20$

\begin{tabular}{|c|c|c|c|c|c|c|c|}
\hline No. & 1 & 2 & 3 & 4 & 5 & 6 & 7 \\
\hline $\begin{array}{r}1 \\
2 \\
3 \\
4 \\
5 \\
6 \\
7 \\
8 \\
9 \\
10 \\
11 \\
12 \\
13 \\
14 \\
15 \\
16 \\
17 \\
18 \\
19 \\
20\end{array}$ & $\begin{array}{l}14 \\
16 \\
16 \\
16 \\
16 \\
16 \\
14 \\
16 \\
16 \\
17 \\
16 \\
17 \\
16 \\
16 \\
16 \\
16 \\
16 \\
16 \\
17 \\
16\end{array}$ & $\begin{array}{l}48 \\
50 \\
50 \\
50 \\
47 \\
51 \\
48 \\
51 \\
48 \\
50 \\
50 \\
53 \\
50 \\
50 \\
51 \\
47 \\
53 \\
50 \\
50 \\
50\end{array}$ & $\begin{array}{l}121 \\
128 \\
132 \\
134 \\
132 \\
134 \\
134 \\
137 \\
132 \\
131 \\
125 \\
128 \\
131 \\
134 \\
131 \\
131 \\
134 \\
125 \\
134 \\
131\end{array}$ & $\begin{array}{l}293 \\
314 \\
312 \\
280 \\
313 \\
319 \\
299 \\
298 \\
314 \\
312 \\
299 \\
314 \\
305 \\
293 \\
300 \\
300 \\
284 \\
289 \\
298 \\
300\end{array}$ & $\begin{array}{l}326 \\
306 \\
306 \\
294 \\
302 \\
320 \\
282 \\
276 \\
287 \\
320 \\
285 \\
299 \\
319 \\
315 \\
278 \\
324 \\
306 \\
313 \\
319 \\
325\end{array}$ & $\begin{array}{l}446 \\
448 \\
440 \\
457 \\
449 \\
454 \\
446 \\
442 \\
451 \\
434 \\
445 \\
448 \\
437 \\
442 \\
437 \\
436 \\
425 \\
412 \\
445 \\
430\end{array}$ & $\begin{array}{l}531 \\
\mathbf{4 9 7} \\
545 \\
522 \\
540 \\
536 \\
536 \\
515 \\
533 \\
522 \\
520 \\
556 \\
531 \\
549 \\
545 \\
545 \\
522 \\
520 \\
483 \\
520\end{array}$ \\
\hline $\begin{array}{l}\text { Mean } \\
2 \text { SD Range } \\
\text { SE } \\
\text { Coeff. Var. }\end{array}$ & $\begin{array}{l}16 \\
14 \cdot 4-17 \cdot 6 \\
0.2 \\
5 \cdot 0\end{array}$ & $\begin{array}{l}49 \cdot 9 \\
46 \cdot 7-53 \cdot 1 \\
0 \cdot 4 \\
3 \cdot 2\end{array}$ & $\begin{array}{l}132 \\
124 \cdot 2-139 \cdot 8 \\
0 \cdot 9 \\
2 \cdot 9\end{array}$ & $\begin{array}{c}301 \cdot 8 \\
280-323 \cdot 6 \\
2.4 \\
3.6\end{array}$ & $\begin{array}{l}305 \cdot 1 \\
272 \cdot 1-338 \cdot 1 \\
3 \cdot 7 \\
5 \cdot 4\end{array}$ & $\begin{array}{l}441 \cdot 2 \\
420 \cdot 2-462 \cdot 2 \\
2 \cdot 3 \\
2 \cdot 4\end{array}$ & $\begin{array}{l}528 \cdot 4 \\
493 \cdot 2-563 \cdot 6 \\
3 \cdot 9 \\
3 \cdot 3\end{array}$ \\
\hline
\end{tabular}




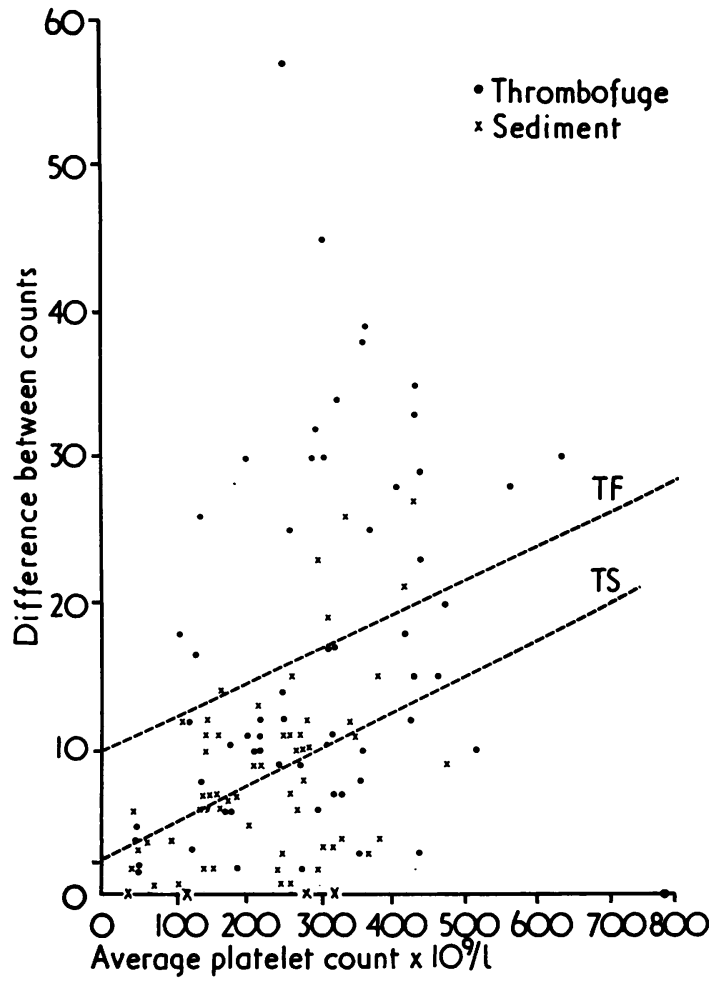

Fig. 4 Duplicate studies: counts performed on platelet-rich plasma prepared by tube sedimentation and Thrombo-fuge. Differences between duplicate counts plotted against average of counts.

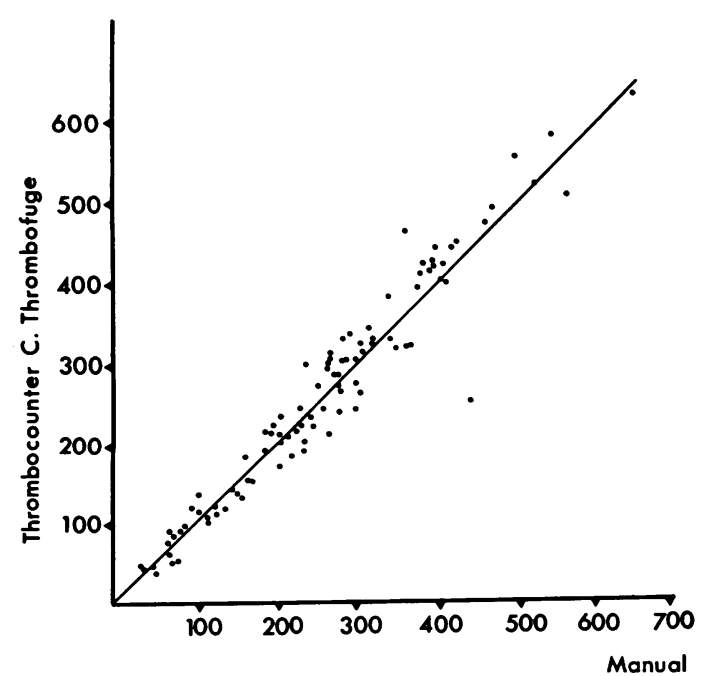

Fig. 5 Comparison of Thrombocounter C/Thrombo-fuge counts with visual counts. the procedure being to count, and then to recount the samples two hours later. Differences between counts were plotted against the average of the two counts (Fig. 4). Differences were never greater than plus or minus $30 \times 10^{9} / 1$ and in general were very much better. Linear regression of the difference plotted against the average gave a small intercept on the $y$ axis. Thus, over the range encountered, differences of approximately $5 \%$ of the average value would be anticipated at the $100 \times 10^{9} / 1$ level and as low as $3 \%$ at the $500 \times 10^{9} / 1$ level. A further 61 samples were counted in duplicate, platelet-rich plasma on this occasion being prepared by the Thrombo-fuge. On this occasion differences between readings plotted against the average showed a greater scatter, the greatest difference recorded being $54 \times 10^{9} / 1$ at the $250 \times 10^{9} / 1$ platelet level. Generally, however, differences did not exceed plus or minus $40 \times 10^{9} / 1$. Linear regression showed a substantial intercept on the $y$ axis. On application of the paired $t$ test, however, the differences were not significant $(t=0.5624 ; \mathrm{n}=61)$.

\section{AGREEMENT WITH LIGHT MICROSCOPY}

In this experiment platelet counts were performed by experienced technicians using the Thrombocounter $\mathrm{C}$ and light microscopy. One hundred and fifteen blood samples were counted following tube sedimentation. The arithmetic mean for instrument counts was $238 \times 10^{9} / 1$ and for visual counts $240 \times 10^{9} / 1$. A further 99 blood samples (Fig. 5) were counted using the Thrombo-fuge. The arithmetic mean for instrument counts was $260 \times 10^{9} / 1$ and for visual counts $254 \times 10^{9} / 1 \quad(r=0.9812$; $\mathrm{n}=$ 99).

Variation in the concentration of EDTA does not lead to discrepant counts (Table 4).

\section{Discussion}

Platelet counting using the Thrombocounter $\mathrm{C}$ with tube sedimentation and Thrombo-fuge methods has been assessed. The system requires preparation of platelet-rich plasma by sedimentation followed by manual dilution before presentation to the instrument for counting. Using tube sedimentation, 30 minutes are required to prepare platelet-rich plasma. With adequate batching of samples the throughput in terms of whole blood platelet counts is 30 per hour. Preparation of platelet-rich plasma by means of the Thrombo-fuge takes 7-8 minutes for 10 samples. Surprisingly, a realistic throughput using this technique is also 30 counts per hour although individual platelet counts can be achieved much more rapidly. These throughput estimates are based on one technician handling the analytical train. 
Table 4 Effect of varying concentrations of EDTA on platelet counts obtained

\begin{tabular}{|c|c|c|c|c|c|}
\hline $\begin{array}{l}\text { Concentration of Sequestrene } \\
(\mathrm{mg} / \mathrm{ml} \text { blood })\end{array}$ & $\begin{array}{l}\text { Manual platelet } \\
\text { count }\left(\times 10^{\circ} / 1\right)\end{array}$ & $\begin{array}{l}S \\
H c t\end{array}$ & $\begin{array}{l}\text { Thro'Counter } C \\
\text { result }\left(\times 10^{\circ} / 1\right)\end{array}$ & Manual Hct & $\begin{array}{l}\text { Thro'Counter } C \\
\text { result }\left(10^{\circ} / I\right)\end{array}$ \\
\hline $\begin{array}{l}5 \cdot 0 \\
3 \cdot 3 \\
2 \cdot 5 \\
2 \cdot 0 \\
1 \cdot 7 \\
1 \cdot 4 \\
1 \cdot 2 \\
1 \cdot 1 \\
1 \cdot 0\end{array}$ & $\begin{array}{l}207 \\
196 \\
201 \\
196 \\
195 \\
208 \\
200 \\
203 \\
200\end{array}$ & $\begin{array}{l}49 \\
50 \\
50 \\
49 \\
49 \\
50 \\
50 \\
50 \\
49\end{array}$ & $\begin{array}{l}212 \\
179 \\
191 \\
218 \\
207 \\
208 \\
180 \\
188 \\
220\end{array}$ & $\begin{array}{l}47 \\
47 \\
48 \\
48 \\
49 \\
49 \\
49 \\
50 \\
50\end{array}$ & $\begin{array}{l}223 \\
195 \\
202 \\
225 \\
207 \\
215 \\
186 \\
188 \\
214\end{array}$ \\
\hline
\end{tabular}

Statistical evaluation proved to be satisfactory. Linearity studies, using both dilution and concentration techniques, gave satisfactory results through a platelet counting range of $20 \times 10^{9} / 1$ to $700 \times 10^{9} / 1$ (Figs. 2 and 3). Carry-over was negligible. Machine drift was not detected. Assessment of instrument precision, determined by series of 20 replicate counts (Tables 2 and 3), resulted in average coefficients of variation for the tube sedimentation method of $4.8 \%$ and for the Thrombo-fuge of $3.7 \%$. These figures show a substantial improvement on the coefficient of variation of $11 \%$ for visual counting previously reported (Brecher et al., 1953). Further evidence of precision was obtained from duplicate counts on individual samples (Fig. 4). Differences between counts were not statistically significant. The accuracy of the Thrombocounter $C$ system was assessed by comparing instrument-rated counts with those obtained using light microscopy, platelet-rich plasma for machine counts being prepared both by sedimentation and by Thrombo-fuge. Again the differences were not statistically significant $(r=0.9812 ; n=99)$. The visual counts by the two technicians were in very close agreement, confirming a previous report (Rowan et al., 1972).

Falsely low and high platelet results have been encountered. Samples from patients with chronic leukaemia who have greatly increased white counts may produce false low results, this being particularly marked in situations where the total white cell count exceeds $50 \times 10^{9} / 1$. The presence of large numbers of giant platelets may also lower the platelet count. Such platelets can obviously settle out, be screened out by the upper threshold setting (although this seems unlikely), or introduce coincidence loss. Elevation of the erythrocyte sedimentation rate produces an unbiased slight increase in scatter of points obtained when graphing machine-rated against visual platelet counts, but this is not of sufficient magnitude to cause concern in routine use. Discrepancies do not occur in platelet counts from patients with paraprotein disturbances. Platelet antibodies, particularly cold agglutinins, may cause false low counts. Spuriously high counts may be obtained due to sample haemolysis, high background counts, instrument electronic malfunction or debris in the orifice, but these may be avoided by careful technique.

Further potential problems can be classified under two headings, namely, those associated with the instrument and those with methodology. The orifice flow time is stable, and problems with electronic malfunction have been minimal. Direct viewing of the orifice is possible through the telescope mounted on the side of the instrument. Local electrical interference can give rise to discrepant counting. Low background counts can be achieved by using prefiltered bulk Isoton. Clotted or haemolysed specimens must be discarded. Diluting and pipetting errors may arise but can be avoided by meticulous technique. The pipetting manoeuvre following the use of the Thrombo-fuge is more difficult than with the tube sedimentation technique. Both systems still rely on mouth pipetting.

\section{References}

Brecher, G., Schneiderman, M., and Cronkite, E. P. (1953). The reproducibility and constancy of the platelet count. American Journal of Clinical Pathology, 23, 15-26.

Bull, B. S., Schneiderman, M. A., and Brecher, G. (1965). Platelet counts with the Coulter Counter. American Journal of Clinical Pathology, 44, 678-688.

Dacie, J. V. and Lewis, S. M. (1975). In Practical Haematology, 5th edition, p. 53. Churchill, London.

Rowan, R. M., Allan, W., and Prescott, R. J. (1972). Evaluation of an automated platelet counting system utilising whole blood. Journal of Clinical Pathology, 25, 218-226. 